\title{
Further characterizations and Helly-property in $k$-trees
}

\author{
H. P. Patil*
}

\begin{abstract}
The purpose of this paper is to obtain a characterization of $k$-trees in terms of $k$-connectivity and forbidden subgraphs. Also, we present the other characterizations of $k$-trees containing the full vertices by using the join operation. Further, we establish the property of $k$-trees dealing with the degrees and formulate the Helly-property for a family of nontrivial $k$-paths in a $k$-tree. We study the planarity of $k$ trees and express the maximal outerplanar graphs in terms of 2-trees and $K_{2}$-neighbourhoods. Finally, the similar type of results for the maximal planar graphs are obtained.
\end{abstract}

Keywords: Trees, Cycles, Paths, Connected graphs, Triangulated graphs, Planar graphs

Mathematics Subject Classification (2010): 05C10

\section{Introduction}

All graphs considered here are finite and simple.For any graph $G$, let $V(G)$ and $E(G)$ denote its vertex set and edge set, respectively. The order of $G$ is $|V(G)|$ and its size is $|E(G)|$. A graph of order $p$ and size $q$ is a $(p, q)$-graph. For any two disjoint graphs $G$ and $H, G+H$ denotes the join of $G$ and $H$. All definitions and notations are not given here may be found in Harary[4]. A graph $G$ is $n$-connected if the removal of any $m$ vertices for $0 \leq m<n$, from $G$ results in neither a disconnected graph nor a trivial graph. 1-connected graphs are simply the connected graphs. A graph $G$ is triangulated if every cycle of length strictly greater than 3 possesses a chord. Any $n$ mutually adjacent vertices i.e., $K_{n}$ in a graph is $n$-clique. For any set $S$ of vertices of a graph $G,\langle S\rangle$ denotes the induced subgraph of $G$ induced by $S$. For

*Department of Mathematics, Pondicherry University; hpppondy@gmail.com

Received: April 2016. Reviewed: May 2016 
any connected graph $G, n G$ denotes the graph with $n$ components, each being isomorphic to $G$.

A family of trees, which are connected and acyclic, can be equivalently defined by the following recursive construction rule:

Step 1. A single vertex $K_{1}$ is a tree.

Step 2. Any tree of order $n \geq 2$, can be constructed from a tree $T$ of order $(n-1)$ by inserting an $n^{\text {th }}$-vertex and joining it to any vertex of $T$.

More general, the multidimensional-trees can be constructed from the above tree-construction procedure by allowing the base of the recursive growth to be any clique. Notice that a connected graph, which is not a tree possesses a tree-like structure, which is actually reflected by constructing the new family of graphs, whose recursive growth just starts from any given clique $K_{k}$. This family of graphs are generally known as $k$-trees or $K_{k}$-trees or $k$-dimensional trees.[1, 5, 7, 8]

Definition 1.1. The family of $k$-trees (or $K_{k}$-trees) is the set of all graphs that can be obtained by the following recursive construction procedure :

1. A clique- $K_{k}$ is the smallest $k$-tree.

2. To a $k$-tree $G$ with $n-1$ vertices for $n \geq k+1$, add a new vertex and make it adjacent to any $k$ mutually adjacent vertices of $G$, so that the resulting $k$-tree is of order $n$.

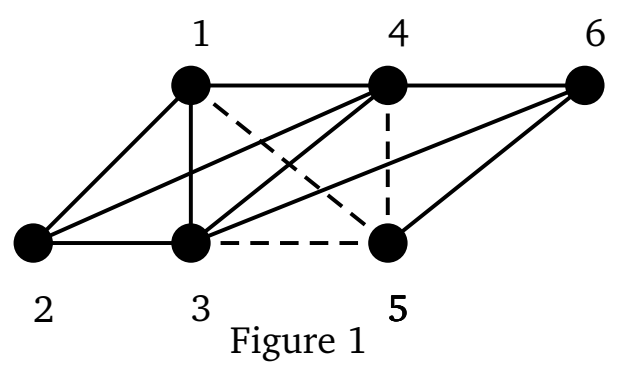

Figure 1 gives the example of a 3 -tree of order 6 . Generally speaking, every $k$-tree $G$ of order $\geq k+1$, can be reduced to a clique $K_{k}$, by sequentially removing the vertices of degree $k$ from $G$.

\section{Properties and Characterizations}

We need the following characterization theorem for later use.

Theorem 2.1. [5] Let $G$ be a ( $p, q)$-graph with $p \geq k+1$. Then $G$ is a $k$ tree if and only if $G$ is $k$-connected, triangulated and either $G$ is $K_{k+2}-$ free or $q=\left(k p-\frac{k(k+1)}{2}\right)$. 
The immediate consequence of Theorem 2.1 is another characterization of $k$-trees in terms of forbidden subgraphs and $k$-connectivity.

Corollary 2.2. Let $G$ be a graph of order at least $k+1$. Then $G$ is a $k$-tree if and only if $G$ is $k$-connected and has no induced subgraph isomorphic to either $C_{n}$ for $n \geq 4$ or $K_{k+2}$.

We first obtain the basic property of $k$-trees dealing with degrees. For this, we need to establish the following lemma.

Lemma 2.3. Every $k$-connected, $(p, q)$-graph $G$ with $p \geq k+1$ and $q=$ $\left(k p-\frac{k(k+1)}{2}\right)$, has at least $k+1$ vertices, whose degrees do not exceed $2 k-1$.

Proof. Since $G$ is $k$-connected, deg $v_{i} \geq k$ for all $v_{i}$ in $V(G)$. Let $t$ be the number of vertices in $G$, whose degrees are at most $2 k-1$. Consequently, $G$ contains $p-t$ vertices of degrees at least $2 k$. Immediately, we have

$\sum_{i=1}^{p} \operatorname{deg} v_{i} \geq t k+(p-t) 2 k$.

On the other hand, by the handshaking theorem, we have

$\sum_{i=1}^{p} \operatorname{deg} v_{i}=2 q=2\left(k p-\frac{k(k+1)}{2}\right)$.

From equations (1) and (2), we have

$$
2 k p-k(k+1) \geq t k+(p-t) 2 k \text {. }
$$

This shows that $t \geq k+1$ and hence, $G$ contains at least $k+1$ vertices, whose degrees do not exceed $2 k-1$.

The direct consequence of Lemma 2.3 is the following result. Moreover, for $k=1$, this result extends the property of trees (Corollary 4.1 (a) p.34, [4]).

Corollary 2.4. Every $k$-tree of order at least $k+1$, has at least $k+1$ vertices, whose degrees do not exceed $2 k-1$.

Proof. Let $G$ be a $k$-tree of order $p \geq k+1$. By Theorem 2.1, $G$ is a triangulated, $k$-connected graph of size $\left(k p-\frac{k(k+1)}{2}\right)$. From Lemma 2.3, the result follows.

Next, we show that the bound given in Corollary 2.4, is the best possible by constructing below a $k$-tree $G$ with exactly $k+1$ vertices, whose degrees do not exceed $2 k-1$. Let $G$ be a graph consists of $K_{k+1} \cup \overline{K_{k+1}}$, with all the possible additional edges $u_{i} v_{j}$ for $i \neq j$, where $u_{i}$ and $v_{j}$ are the vertices in $K_{k+1}$ and $\overline{K_{k+1}}$, respectively (for $1 \leq i, j \leq$ $k+1$ ). Now, we observe that $G$ is a $k$-tree of order $2 k+2$ and it contains $k+1$ vertices of degree $k$ and $k+1$ vertices of degree $2 k$. 
Definition 2.5. Let $G$ be a graph of order $p$. A vertex $v$ in $G$ is called a full-vertex if $\operatorname{deg} v=p-1$.

For example, $K_{k}+\overline{K_{p-k}}$ (for $k<p$ ), is a $k$-tree of order $p$, containing exactly $k$ full-vertices. We now obtain a characterization of $k$-trees containing at least one full-vertex.

Theorem 2.6. Let $G$ be a graph of order $p \geq k+1$. Then $G$ is a $k$-tree containing a full-vertex if and only if $G$ is isomorphic to $K_{1}+H$, where $H$ is $a(k-1)$-tree of order $p-1$.

Proof. Suppose that $G$ is a $k$-tree, containing a full-vertex $v$. By Theorem 2.1, $G$ is a $k$-connected, triangulated graph of size $\left(k p-\frac{k(k+1)}{2}\right)$. Let $\langle\{v\}\rangle \cong K_{1}$. Since $\operatorname{deg} v=p-1$ in $G$, the removal of $v$ from $G$ certainly reduces its connectivity by one, without affecting its triangularity property and further, we have

$$
|E(G-v)|=\left(k p-\frac{k(k+1)}{2}\right)-(p-1)=(k-1)(p-1)-\frac{k(k-1)}{2} .
$$

From Theorem 2.1, $G-v$ is a $(k-1)$-tree of order $p-1$. However, we see that $G$ is isomorphic to $K_{1}+(G-v)$.

Conversely, assume that $G$ is isomorphic to $K_{1}+H$, where $H$ is a $(k-1)$-tree of order $p-1$. Since $\operatorname{deg} v=p-1$ in $G$, it follows that $H$ is isomorphic to $G-v$. Consequently, $G=K_{1}+(G-v)$ is a $k$-connected, triangulated graph of size $\left(k p-\frac{k(k+1)}{2}\right)$. By Theorem 2.1, $G$ is a $k$-tree.

Repeated application of Theorem 2.6, yields the general criterion for $k$-trees containing at most $k$ full-vertices.

Corollary 2.7. Let $G$ be a graph of order $p \geq k+1$. Then $G$ is a $k$-tree containing $t$ full-vertices $(1 \leq t \leq k)$ if and only if $G$ is isomorphic to $K_{t}+T_{p-t}$, where $T_{p-t}$ is a $(k-t)$-tree of order $p-t$ and $T_{p-k}$ is a forest.

\section{Helly-property on $k$-paths}

We begin with the notion of $m$-walk for $m \geq 2$, which extends the concept of a walk (i.e., 1-walk) introduced by Beineke and Pippert.[1]

Definition 3.1. (1). A $m$-walk for $m \geq 1$, in a graph $G$, denoted by $W\left(K_{m}^{0}, K_{m}^{n}\right) ; n \geq 0$, is an alternating finite sequence of its distinct cliques $K_{m}$ and $K_{m+1}$ of the form:

$\left(K_{m}^{0}, K_{m+1}^{1}, K_{m}^{1}, K_{m+1}^{2}, \ldots, K_{m}^{n-1}, K_{m+1}^{n}, K_{m}^{n}\right)$, beginning and ending with the cliques $K_{m}^{0}$ and $K_{m}^{n}$, respectively such that for each $i(1 \leq i \leq n), K_{m+1}^{i}=$ $K_{m}^{i-1} \cup K_{m}^{i}$ and $K_{m}^{i-1} \cap K_{m}^{i}=K_{m-1}$.

(2). A $m$-walk $W\left(K_{m}^{0}, K_{m}^{n}\right) ; n \geq 0$, is called a m-path if all its cliques 
$K_{m}^{0}, K_{m}^{1}, \ldots, K_{m}^{n}$ and $K_{m+1}^{1}, K_{m+1}^{2}, \ldots, K_{m+1}^{n}$ are distinct. The length of a $m$-path, is the number of occurrences of cliques $K_{m+1}$ in it. For example, any clique $K_{m}$ is a trivial $m$-path ; $K_{m+1}$ is a nontrivial $m$-path of length $1 ; K_{m}+\overline{K_{2}}$ is a nontrivial m-path of length 2 .

In Figure 2, the anatomy of a 2-path is shown.

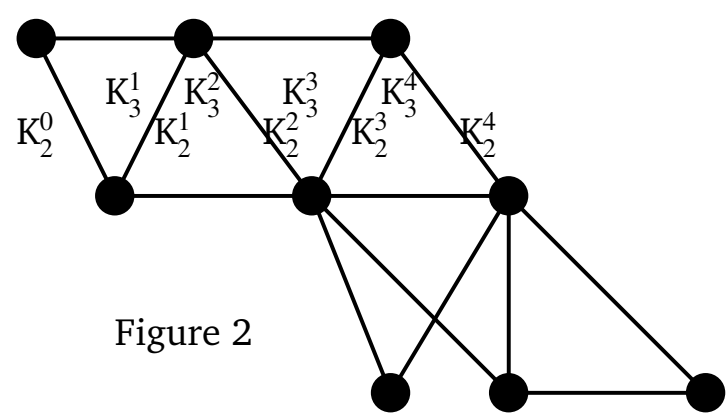

Let $\Pi=\left\{J_{i}: i \in I\right\}$ be a family of subsets of a finite set $S$ (where $I$ denotes the index set). Then $\Pi$ is said to satisfy the Helly-property if $J_{i} \cap J_{j} \neq \emptyset$ for all $i, j$ in $I$, implies that $\cap_{k \in I} J_{k} \neq \emptyset$.

For example, $\Pi=\left\{J_{1}, J_{2}, J_{3}\right\}$, where the nontrivial paths : $J_{1}=$ $a b c ; J_{2}=c b d ; J_{3}=a b d$, of the tree $K_{1,3}$ as shown in Figure 3.

Notice that every two paths in $\Pi$ have a nontrivial intersection, but there is no common nontrivial path for all three paths in $\Pi$.

We now establish the Helly-property for a family of nontrivial $k$ paths of a $k$-tree.

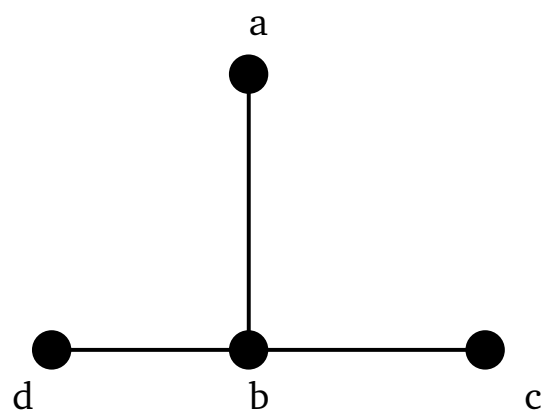

Figure 3

Proposition 3.2. Let $\Pi=\left\{J_{i}: i \in I\right\}$ be a finite family of nontrivial $k$-paths of a $k$-tree. If every three $k$-paths $J_{i}, J_{j}, J_{k}$ for $i, j, k \in I$, have a nontrivial intersection, then $\cap_{n \in I} J_{n}$ is a nontrivial intersection.

Proof. Let $G$ be a $k$-tree. We prove the result by induction on the number of nontrivial $k$-paths of $G$. Assume that $\cap_{n \in J} J_{n}$ is isomorphic to $W$, 
where $|J|=t<|I| ; J$ is an index set, is a nontrivial $k$-path of $G$.

If $J_{t+1}$ has no nontrivial intersection with $W$, then there exist always three $k$-paths $J_{t+1}, J_{t}$ and $J_{t-1}$ of $G$, which have no nontrivial intersection. (In fact, for $k=1$, this fact is illustrated in Figure 4). This is a contradiction to the hypothesis. Hence, the desired property is proved.

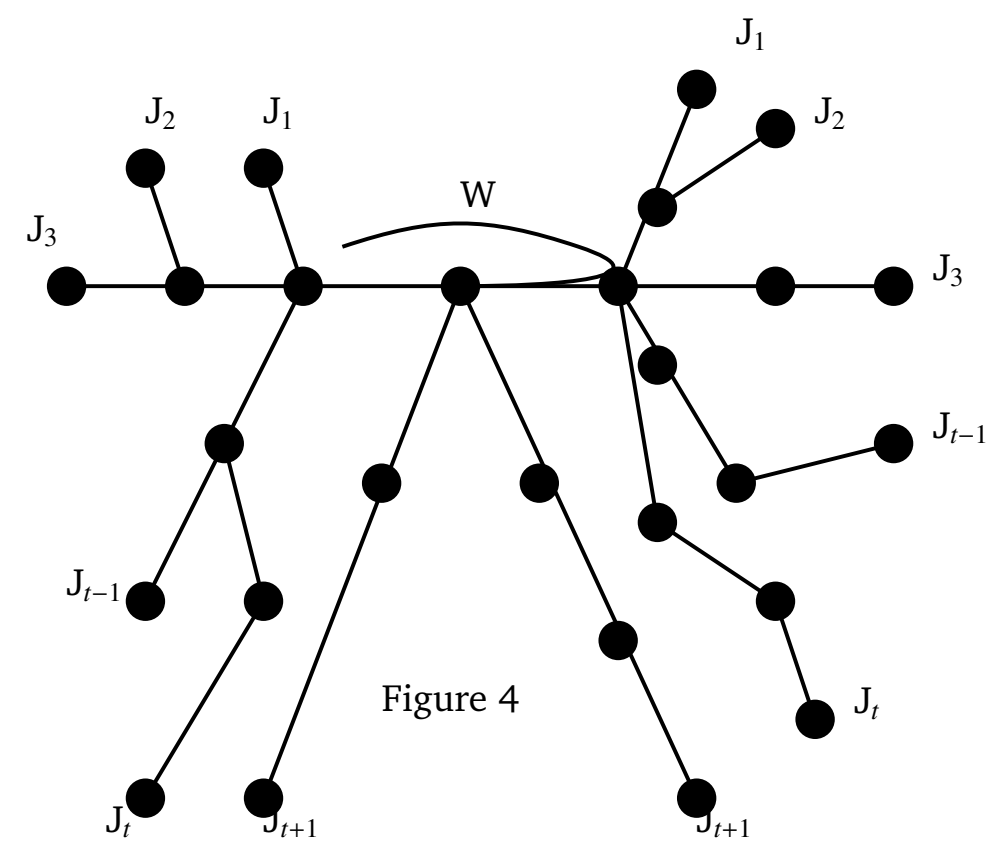

\section{Planarity and Clique-neighbourhoods}

The neighbourhood of a vertex $u$ in a graph $G$ is the set $N(u)$ consisting of all the vertices, which are adjacent to $u$. A vertex $u$ is simplicial if $N(u)$ induces a clique in $G$.

Definition 4.1. For any clique $K_{p}$ of a graph $G$ with vertices $u_{1}, u_{2}, u_{3}, \ldots, u_{p}$, the $K_{p}$-neighbourhood, denoted by $N\left(K_{p}\right)$ is $\cap_{i=1}^{p} N\left(u_{i}\right)$.

Notice that 1-trees (i.e., trees) are obviously planar. The maximal outerplanar graphs are the special class of 2-trees. The triangulated, maximal planar graphs are restricted family of 3-trees. All nontrivial 4-trees (other than $\left.K_{4}\right)$ and $k$-trees $(k \geq 5)$ are nonplanar. To study (outer)planarity, let us first establish the following lemma.

Lemma 4.2. Let $G$ be a $k$-tree of order $\geq k+1$. For any clique $K_{k}$ in $G$, a). $N\left(K_{k}\right) \neq \emptyset$.

b). $N\left(K_{k}\right)$ is an independent set. 
Proof. To prove (a), we use the induction on order $p \geq k+1$ of $G$. If $p=k+1$, then $G=K_{k+1}$. Obviously, $\left|N\left(K_{k}\right)\right|=1$ for any clique $K_{k}$ in $G$ and hence the result is obvious. We assume that the result holds for any $p: k+2 \leq p \leq n$. Let $G$ be a $k$-tree with $p=n+1$. Then by Definition 1.1, $G$ contains a simplicial vertex $u$ of degree $k$ and $G-u$ is a $k$-tree of order $n$. By induction hypothesis, $N\left(K_{k}\right) \neq \emptyset$ for any clique $K_{k}$ in $G-u$. Let $N(u)=\left\{u_{1}, u_{2}, \ldots, u_{k}\right\}$ and $N(u)$ is isomorphic to $K_{k}$. Consider any clique $K_{k}^{i}$ of $G$ with $V\left(K_{k}^{i}\right)=\{u\} \cup\left(N(u)-\left\{u_{i}\right\}\right)$ for $1 \leq i \leq k$. Immediately, we observe that $N\left(K_{k}^{i}\right)=\left\{u_{i}\right\}$. Thus, $N\left(K_{k}^{i}\right) \neq \emptyset$. By induction, the result follows for all $p \geq k+1$.

To prove (b), if possible, we assume that for some clique $K_{k}$ in $G$, $N\left(K_{k}\right)$ is not independent. Then $G$ contains at least two vertices $u$ and $v$ in $N\left(K_{k}\right)$ such that $u$ and $v$ are adjacent in $G$. This shows that $\langle N(u) \cup\{u, v\}\rangle$ is isomorphic to $K_{k+2}$ in $G$. This is not possible (by Theorem 2.1), because $G$ is a $k$-tree.

In [5], it is proved that any graph $G$ of order $\geq 3$, is maximal outerplanar if and only if $G$ is 2-connected, triangulated and outerplanar. Next, we present another characterization of a maximal outerplanar graph involving 2-trees and $K_{2}$-neighbourhoods.

Proposition 4.3. Let $G$ be a graph of order $\geq 3$. Then $G$ is maximal outerplanar if and only if $G$ is a 2-tree and for any complete graph $K_{2}$ of $G,\left\langle N\left(K_{2}\right)\right\rangle$ is either $K_{1}$ or $2 K_{1}$.

Proof. Suppose that $G$ is maximal outerplanar. Immediately, $G$ is 2connected, triangulated and outerplanar. Since $G$ is outerplanar, $G$ is $K_{4}$-free. By Theorem 2.1 with $k=2, G$ is a 2-tree. On contrary, assume that $\left|N\left(K_{2}\right)\right| \geq 3$ for some complete graph $K_{2}$ of $G$. Let $x, y$ and $z$ be the vertices in $N\left(K_{2}\right)$. Consequently, $\langle\{u, v, x, y, z\}\rangle$ isomorphic to $K_{2}+3 K_{1}$ appears in $G$. But $K_{2}+3 K_{1}$ contains a subgraph isomorphic to $K_{2,3}$ and hence $G$ is not outerplanar. This leads to a contradiction. So, $\left|N\left(K_{2}\right)\right| \leq 2$ for each complete graph $K_{2}$ of $G$. From Lemma 4.1 with $k=2$, we have $\left|N\left(K_{2}\right)\right| \geq 1$ and $\left\langle N\left(K_{2}\right)\right\rangle$ is either $K_{1}$ or $2 K_{1}$. Necessity is thus proved.

It is easy to prove the converse.

The immediate consequence of the above proposition is Corollary 11.9 (a) of [4, p. 107]. Certainly, this bound can be improved for nonouterplanar, 2-trees.

Corollary 4.4. Every 2-tree other than maximal outerplanar, has at least three vertices of degree 2.

Proof. Follows from the immediate consequence of Proposition 4.3. 
Notice that a maximal planar graph need not be triangulated. For example, $C_{4}+2 K_{1}$ is maximal planar but not triangulated.

Proposition 4.5. Let $G$ be a triangulated graph of order $\geq 4$. Then $G$ is maximal planar if and only if $G$ is a 3-tree and for any triangle $K_{3}$ in $G,\left\langle N\left(K_{3}\right)\right\rangle$ is either $K_{1}$ or $2 K_{1}$.

The proof follows on the similar arguments as used in the proof of Proposition 4.3, by using Theorem 2.1 with $k=3$. result.

The following corollary is the immediate consequence of the above

Corollary 4.6. Every nonplanar 3-tree, has at least three vertices of degree 3.

\section{Acknowledgement}

The research was supported by UGC-SAP (DRS-II).

\section{References}

[1] L. W. Beineke and R. E. Pippert, "Properties and characterizations of k-trees," Mathematica, vol. 18, pp. 141-151, 1971.

[2] M. Borowiecki and H. P. Patil, "On colouring and the chromatic polynomial of k-trees," J. Comb. Inf. and Syst. Sci., vol. 11, pp. 124-128, 1986.

[3] C.Y. Cho, N.Z. Li and S.J. Xu, "On $q$-trees," J. Graph Theory, vol. 10, pp. 129136, 1986.

[4] F. Harary, Graph Theory. Reading, MA: Addison-Wesley, 1969.

[5] H. P. Patil, "On the structure of $k$-trees," J. Comb. Inf. and Syst. Sci., vol. 11, pp. 57-64, 1986.

[6] H. P. Patil, "A relationship between $n$-degenerate graphs, uniquely colourable graphs and $k$-trees," Proc. Symp. on Graph Theory and Combinatorics, Kochi, Kerala, India, 17-19 May 1991, pp. 93-97.

[7] H. P. Patil, "Studies on $k$-trees and some related topics," Ph.D. dissertation, Univ. Warsaw, Poland, 1984.

[8] D. J. Ross, "On simple characterizations of $k$-trees," Discrete Math., vol. 7, pp. 317-322, 1974. 